\title{
Design Active Intelligent Multi Degrees of Freedom Joint Controller for Dental Automation
}

\author{
Maryam Rahmani, Nasim Sobhani, Hamid Cheraghi Farzin Piltan and Farzin \\ Matin \\ Intelligent System and Robotic Lab, Iranian Institute of Advanced Science and \\ Technology (IRAN SSP), Shiraz/Iran, www.iranssp.org/english \\ piltan_f@iranssp.org
}

\begin{abstract}
Following the developments in industrial robot technology, robotics has found its way into the medical field and is used in a range of surgical disciplines. The main purpose of the use of robots is to increase the precision, quality and safety of surgical procedures. Robotics is not yet used in dentistry even though all the necessary technologies have already been developed and could easily be adapted. Some of the technologies are already used in dentistry, such as image-based simulation of implant surgery followed by the use of surgical guides, and creating digital impressions of pre parathions using an intra-oral scanner, after which a milling device produces the restoration, but we have not yet seen any robot able to prepare teeth for crowns, inlays or bridges. Such a robot would fundamentally be a dental drilling device coupled with a navigation device to determine the correct position of the device in relation to the patient. However this technique is very essential to have the minimal pain and reduce bleeding but control of these robots/joints are very complicated.

This paper proposes new fuzzy type sliding mode controller for multi-DOF joints to control of motor vibration. The fuzzy $(P D+I)^{2}$ single-input single-output (SISO) fuzzy system is used to control of chattering in sliding mode controller. In most research al researchers wants to eliminate the chattering but, this research focuses on chattering control. Various operation situations such as the set point control and the trajectory control are simulated. The simulation results demonstrate that the chattering and the steady state errors, which usually occur in the classical sliding mode control, are limitation control and satisfactory trajectory tracking is achieved.
\end{abstract}

Keywords: sliding mode controller, Dental robot manipulator, dental automation, fuzzy logic controller, vibration control, multi degrees of freedom joint

\section{Introduction and Background}

One of the best ways to maintenance of comprehensive oral health is Nanodentistry. It is employing nanomaterials, biotechnology, including tissue engineering and ultimately, dental nanorobotics. Nanodentistry can do oral health maintenance using mechanical dentifrobots, like local anesthesia, dentition renaturalization, permanent hypersensitivity cure, complete orthodontic realignments, covalently bonded diamond dised enamel and etc. Any product containing nano particles are Nano products that can be made by combining atomic elements to create mechanical nanoscale objects. Dentin hypersensitive of natural teeth have higher surface density of dentinal tubules and diameter and also larger than nonsensitive teeth. In dental nanorobots, we can use native biological materials, so it could selectively occlude specific tubules in a few minutes and offering patients a quick cure. Even Orthodontic nanorobots could directly effect to the periodontal ligaments, and allowing rapid and painless tooth alignment in correct 
positioning within minutes to hours. Nanorobotic manufacture of a biologically auto logous whole replacement tooth, that is, 'complete dentition replacement therapy' should become conveniently of a typical office visit with use of a desktop manufacturing facility, which would invent or fabricate the new tooth in the dentist's office not in the laboratory.

In Nanorobotic analgesics, don't use needles, so there is a greater ability to control the analgesic effect, fast and reversible with avoid of side effects, to give patient comfort and also reduced nervousness. Nanorobotic dentifrice (dentifrobots) take by mouthwash or toothpaste that could control all supragingival and sub gingival surfaces using once a day or more, metabolizing the organic matter into harmless and odorless vapors and prevent calculus debridement and plaque accumulation. With this kind of daily dental care available from an early age, we can prevent tooth decay and gingival diseases [1-5].

Robots are typically thought to be used for industrial purposes however they are beginning to gain the attention of the medical field. Telemanipulator is collection of links that connect by joints, these joints can be revolute, prismatic and spherical, that revolute joint has rotary motion around an axis, prismatic joint has linear motion around an axis and spherical joints move in three axes. Revolute and spherical joints provide one degree of freedom (DOF) but spherical joints provide three DOF [6-7]. Multi-degree-offreedom (DOF) actuators are finding wide use in a number of Industries. Currently, a significant number of the existing robotic actuators that can realize multi-DOF motion are constructed using gear and linkages to connect several single-DOF motors in series and/or parallel. Not only do such actuators tend to be large in size and mass, but they also have a decreased positioning accuracy due to mechanical deformation, friction and backlash of the gears and linkages. A number of these systems also exhibit singularities in their workspaces, which makes it virtually impossible to obtain uniform, high-speed, and high-precision motion. For high precession trajectory planning and control, it is necessary to replace the actuator system made up of several single-DOF motors connected in series and/or parallel with a single multi-DOF actuator. The need for such systems has motivated years of research in the development of unusual, yet high performance actuators that have the potential to realize multi-DOF motion in a single joint. One such actuator is the spherical motor. Compared to conventional robotic manipulators that offer the same motion capabilities, the spherical motor possesses several advantages. Not only can the motor combine 3-DOF motion in a single joint, it has a large range of motion with no singularities in its workspace. The spherical motor is much simpler and more compact in design than most multiple single-axis robotic manipulators. The motor is also relatively easy to manufacture [8-10].

Surgery is a medical procedure involving an incision in the human body performed to repair damage or arrest disease. In a fully invasive operation, an open incision is made that is large enough for the surgeon to view the internal organs and perform the operation. For example, in open-heart surgery a long incision is made along the sternum, after which the sternum is split and retracted (median sternotomy). These open incisions increase trauma to a patient beyond what is experienced from the actual repair. After the operation, the patient must heal from trauma associated with the repaired organs and from the open incision. This results in long recovery times and opportunities for infection [3]. Endoscopic Surgery, also called Minimally Invasive Surgery (MIS), is a type of operation that has been developed to reduce trauma associated with making these large open incisions. This type of operation involves viewing the operational field on a television monitor by inserting a special camera, called an endoscope, through a small incision in the skin. To perform the operation, "long, thin, manually operated instruments" are inserted through other small incisions called trocars [3]. This type of surgery reduces the size of the open incisions and therefore results in less pain and scarring after surgery, faster recovery times, and less risk of infection [4].

A dental industry does have benefits over fully invasive surgery but there are also several disadvantages associated with performing this type of operation. The two basic 
disadvantages involve viewing the internal operational field and the dental tool interface. Using a dental camera, the operational field is viewed on a 2-D television monitor providing only a limited view with no depth perception. Advances in visual technology have provided surgeons with equipment that allow them to view the field in 3-D however this technology is expensive [5]. Another disadvantage is that the dental operation is operated by a dental assistant. This requires the surgeon to communicate motion instructions which becomes difficult when giving instructions such as how far to move the tools and in which direction. It has also been reported that small tremors from the scope-holding assistant, magnified onto the television monitor, can cause nausea among the dental team [5]. Disadvantages associated with the dental tool interface involve the dental tools and the way that the operation is performed. Often the dental tools are heavy, lacking ergonomic design, and do not have the same DOF/dexterity as a human hand. As mentioned before, these tools are inserted through small incisions in the patient to perform the operation. This creates the fulcrum/lever effect whereas the surgeon is required to transpose each hand motion to get the desired internal motion. For example, if it is desired to move the tool manipulator to the left within the patient the surgeon must transpose this motion in his mind and move the tool handle to the right. Dentists must also deal with the amplification of tremors in their hands due to the fulcrum/lever effect and the length of the tools [3]. Performing an operation through small incisions using dental tools removes the ability for a surgeon to use the sense of touch to gain more information about the internal tissue (haptic feedback). Tools that provide surgeons with this type of information are being developed, however they are not yet in use. Dentists are at risk for musculoskeletal disorders because of lower job control and working long hours. The vascular, neurologic, and osteoarticular disorders in the upper limbs of vibration-exposed workers (like dentists) is called hand-arm vibration syndrome. Also there is a risk for wrist osteoarthritis and for elbow arthritis and osteophytosis in workers exposed to shocks and low-frequency vibration of high magnitude tools that it increased occurrence of digital vasospastic disorders called vibration-induced white finger (VWF) and also hand neuropathy has been reported caused by use of high and low speed tools with high frequency vibration by working with high speed grinding machines that exposed to high frequency vibration up to 40khz. Epidemiologic studies show the increased risk of carpal tunnel syndrome among vibration exposed workers. Low frequency vibration is transmitted from the area of skin that contact with the tool and might produced nerve damaged through swelling of the endoneurium and impaired microcirculation, but with high frequency vibration the mechanical energy is absorbed in the skin so there is no proximal nerve damage accrue. The most important goal among researchers are prevention of hand/wrist complaints. Figure 1 shows the dental robot manipulator.

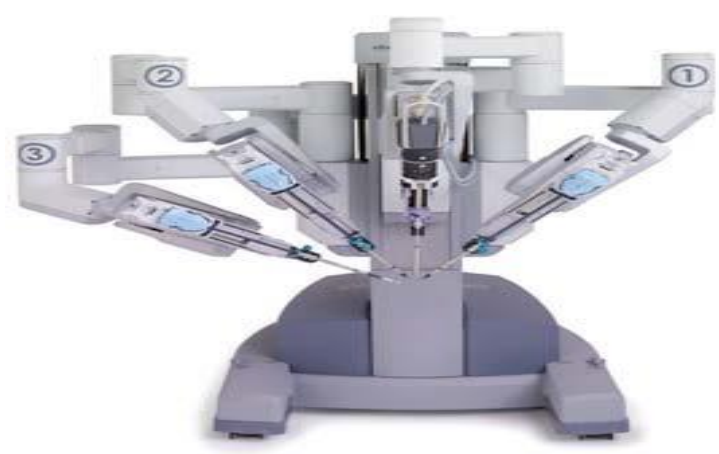

Figure 1. Dental Robot Manipulator 
In the late 1980's, researchers motivated by the limitations of dental surgery turned to robotic technology to make improvements [8-9]. Although the idea of using robotics for telepresence surgery was not a new idea at this time, research and development into this concept rapidly progressed around the success of MIS techniques and became possible with advancements in computing power [10]. By 1998, two major companies had developed surgical systems approved by the FDA for commercial use. Computer Motion in Goletta, California developed the Zeus Surgical System and Intuitive Surgical in Sunnyvale, California developed the daVinci Surgical System. Currently only the daVinci is in production due to the merger of Computer Motion and Intuitive Surgical in 2003.

Classical sliding mode control (CSMC) is a powerful scheme for nonlinear systems with uncertainty [8-10]. This control scheme suffers from some problems, however. In order to guarantee the stability of the sliding mode system, the boundary of the uncertainty has to be estimated. A large value has to be applied to the control gain when the boundary is unknown. Unfortunately, this large control gain may cause chattering on the sliding surface and therefore deteriorate the system performance. Several approaches for reducing the chattering have been proposed, among which the well known one is to apply a saturation function [11] to the control gain when the sliding surface is within a boundary of the sliding hyper-plane. This approach, however, does not guarantee the convergence of the output. In other words, there exists a nonzero steady-state error in the output. An alternative way to solve the chattering problem is the application of the fuzzy logic [12] in the construction of the control input. Fuzzy logic has proven to be a potent tool in the sliding mode control of time-invariant linear systems [13-22] as well as timevarying nonlinear systems [14]. It provides methods for formulating linguist rules from expert knowledge and is able to approximate any real continuous system to arbitrary accuracy. Thus, it offers a simple solution dealing with the wide range of the system parameters. In recent years, artificial intelligence theory has been used in nonlinear control systems. Neural network, fuzzy logic and neuro-fuzzy are synergically combined with nonlinear classical controller and used in nonlinear, time variant and uncertain system. Fuzzy logic controller (FLC) is one of the most important applications of fuzzy logic theory. This controller can be used to control nonlinear, uncertain, and noisy systems. This method is free of some model techniques as in model-based controllers. As mentioned that fuzzy logic application is not only limited to the modelling of nonlinear systems but also this method can help engineers to design a model-free controller. Control of nonlinear system using model-based controllers is based on system dynamic model. These controllers often have many problems for modelling. Conventional controllers require accurate information of dynamic model of system, but most of time these models are MIMO, nonlinear and partly uncertain therefore calculate accurate dynamic model is complicated. The main reasons to use fuzzy logic methodology are able to give approximate recommended solution for uncertain and also certain complicated systems to easy understanding and flexible. Fuzzy logic provides a method to design a model-free controller for nonlinear plant with a set of IF-THEN rules. Mohan and Bhanot have addressed comparative study between some adaptive fuzzy and a new hybrid fuzzy control algorithm for manipulator control. They found that self-organizing fuzzy logic controller and proposed hybrid integrator fuzzy give the best performance as well as simple structure [18-22].

In this research the new technique of fuzzy $(\mathrm{PD}+\mathrm{I})^{2}$ sliding mode controller is used to chattering limitation the effect of motor vibration. This paper is organized as follows;

- Section 2, is served as a modeling and formulation of spherical joint.

- section 3, introduces and describes the control design for reduce the motor vibration. 
- Section 4 presents the simulation results and discussion of this algorithm applied to a spherical joint.

- the final section (section 5), describe the conclusion.

\section{Theorem:}

\section{Case Study (Dynamic Formulation of Robot Manipulator)}

Case Study (Dynamic of Spherical Joint): Medical robots are tools that are being used by doctors to increase there capabilities and abilities to diagnose and cure diseases. They are not replacing doctors, but they are providing them with ways to perform better. Medical robots use range from autonomously performing a specific task during an operation to providing a human-machine interface that helps perform the entire procedure. There are several commercial companies that offer these systems however they are not being widely used. Some of the reasons for this revolve around questions regarding there effectiveness, safety, and cost [9]. Before talking more specifically about the different types and tasks of medical robots, it will help to give a definition of a robotic system. A robot is a mechanical system that is capable of performing a physical task [10]. It can be broken down into three main components: a control device, actuators and mechanical parts, and sensors. These three components allow a robot to interact with its environment. The controller is the brains of the system processing information and changing the actuators and mechanical parts based on this information. The actuators and mechanical parts provide the actual motion of the robot system. The sensors of the system allow the robot to get feedback on its position and in smarter systems data from the environment. Benefits of using robotics are that they can perform motions with very high precision repetitively without fatigue. They are capable of performing tasks that are not possible by humans such as lifting heavy objects and can hold a very precise position endlessly. From these benefits it is not hard to see why research is being done to implement robotics into some of the very difficult, fatiguing, and precise procedures that doctors/surgeons are performing. Medical robots can be divided according to their level of autonomy into three categories: active, semiactive, and passive [6-7]. Active medical robots autonomously perform a specific task during an operation that was programmed prior to the procedure. These robots take on an active role of performing a task under the supervision of the operator. Using this type of robot typically involves three steps: preoperative planning, program verification, and performing the operation. Semiactive medical robot takes on some of the features of both the passive and active medical robots. Similar to a passive robot, a surgeon will directly control the robotic system however the system will provide some type of constraint. This type of medical robot has been classified as having constrained cooperative control autonomy [9]. Passive medical robots are systems that perform motions only under the command of a human operator through some interface (joystick, foot pedal, etc.). These robots are also called surgical assistants, surgical extenders, and telemanipulators [9]. These robots are programmed to listen to external interfaces for motion commands and other tasks. As opposed to active medical robots, there is no preoperative motion commands programmed into the controller. Most of these robots are used for dental procedures were they may perform the task of positioning a dental camera or actually manipulate the dental tools under a surgeon's control. The robotic surgical system described in this research is a tool that would assist a surgeon in performing a dental procedure. It does this by holding and physically manipulating an dental tool under the direct control of the surgeon. It should be noted that the robot does not perform the surgery, but only provides assistance by manipulating the tools position and manipulator at the surgeons command. The system is classified as a passive medical robot, more specifically as a master-slave telemanipulator. In this type of system, the master has direct control over all movements of the robotic 
system. This is an essential feature for a surgical robot because only the surgeon should have the ability to move the robot arm. In between the surgeon and the Robotic System is a human machine interface that provides the surgeon with control of the system.

Since its inception, the field of multi-DOF actuator dynamics has presented many issues in refining both theory and operations; one of the most challenging areas of study has been the problem of computational efficiency in the dynamics of mechanisms. Many efficient algorithms in dynamics have been developed to address this problem.

Dynamic equation is the study of motion with regard to forces. Dynamic modeling is vital for control, mechanical design, and simulation. It is used to describe dynamic parameters and also to describe the relationship between displacement, velocity and acceleration to force acting on multi-DOF actuators. To calculate the dynamic parameters which introduced in the following lines, four algorithms are very important.

i. Inverse Dynamics, in this algorithm, joint actuators are computed (e.g., force/torque or voltage/current) from end-effector position, velocity, and acceleration. It is used in feed forward control.

ii. Forward Dynamics used to compute the joint acceleration from joint actuators. This algorithm is required for simulations.

iii. The Joint-space Inertia Matrix, necessary for maps the joint acceleration to the joint actuators. It is used in analysis, feedback control and in some integral part of forward dynamics formulation.

iv. The Operational-space Inertia Matrix, this algorithm maps the task accelerations to task actuator in Cartesian space. It is required for control of endeffector.

Several different methods are available to compute multi-DOF actuators dynamic equations. These methods include the Newton-Euler (N-E) methodology, the LagrangeEuler (L-E) method, and Kane's methodology [5].

The Newton-Euler methodology is based on Newton's second law and several different researchers are signifying to develop this method [5-8]. This equation can be described the behavior of a multi-DOF actuators joint-by-joint from base to endeffector, called forward recursion and transfer the essential information from end-effector to base frame, called backward recursive.

The literature on Euler-Lagrange's is vast but a good starting point to learn about it is in[9-10]. Calculate the dynamic equation multi-DOF actuators using E-L method is easier because this equation is derivation of nonlinear coupled and quadratic differential equations.

The Kane's method was introduced in 1961 by Professor Thomas Kane[9]. This method used to calculate the dynamic equation of motion without any differentiation between kinetic and potential energy functions.

The dynamics of multi-DOF actuators illustrate the relationship between force and motion. The generalized force for a multi-DOF actuator can be described as a secondorder nonlinear differential equation. The dynamic equation of multi-DOF actuators is derived using the Lagrangian. The Lagrangian is derived by subtracting potential energy from kinetic energy.

The dynamic equation of multi-DOF actuator governed by the following equation [1721]:

$H(q)\left[\begin{array}{c}\ddot{\alpha} \\ \ddot{\beta} \\ \ddot{\gamma}\end{array}\right]+B(q)\left[\begin{array}{c}\dot{\alpha} \dot{\beta} \\ \dot{\alpha} \dot{\gamma} \\ \dot{\beta} \dot{\gamma}\end{array}\right]+C(q)\left[\begin{array}{c}\dot{\alpha}^{2} \\ \dot{\beta}^{2} \\ \dot{\gamma}^{2}\end{array}\right]=\left[\begin{array}{c}\tau_{x} \\ \tau_{y} \\ \tau_{z}\end{array}\right]$

- $\tau$ is actuation torque

- $\quad H(q)$ is a symmetric and positive define inertia matrix

- $\mathrm{B}(\mathrm{q})$ is the matrix of coriolios torques

- $\mathrm{C}(\mathrm{q})$ is the matrix of centrifugal torques. 
The angular acceleration is found as to be:

$$
\left[\begin{array}{c}
\ddot{\alpha} \\
\ddot{\beta} \\
\ddot{\gamma}
\end{array}\right]=H^{-1}(q) \cdot\left\{\tau-\mathbf{B}(\boldsymbol{q})\left[\begin{array}{c}
\dot{\alpha} \dot{\beta} \\
\dot{\alpha} \dot{\gamma} \\
\dot{\boldsymbol{\beta}} \dot{\gamma}
\end{array}\right]-C(q)\left[\begin{array}{c}
\dot{\alpha}^{2} \\
\dot{\boldsymbol{\beta}}^{2} \\
\dot{\gamma}^{2}
\end{array}\right]\right\}
$$

Dental Robot Platform: The robotic dental system described in this research is a tool that would assist a dentist in performing a drilling procedure. It does this by holding and physically manipulating a dentistry tool under the direct control of the dentist. It should be noted that the robot does not perform the dentistry, but only provides assistance by manipulating the tools position and manipulator at the dentists command. The system is classified as a passive medical robot, more specifically as a master-slave telemanipulator (see Figure 2). In this type of system, the master has direct control over all movements of the robotic system. This is an essential feature for a dental robot because only the dentist should have the ability to move the robot arm. In between the dentist and the Robotic System is a human machine interface that provides the dentist with control of the system.

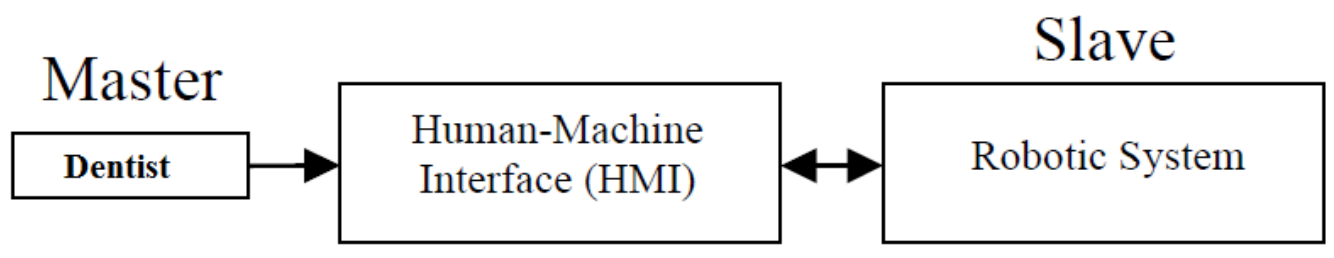

Figure 2. Master-Slave Telemanipulator

The human-machine interface (HMI) is the instruments provided by which the surgeon can control the robotic system to perform an operation. It is composed of a computer (monitor, cpu, keyboard, mouse). The two joysticks provide direct control of the robotic system's movements. The first joystick is used to control the robotic arm while the second joystick controls the dental manipulator. The robotic arm joystick provides XYZ motion control of the drilling end effecter in reference to the world frame. Joystick motions along the $\mathrm{X}$ and $\mathrm{Y}$ axis are converted to $\mathrm{X}$ and $\mathrm{Y}$ displacements while rotation of the joystick is converted to $\mathrm{Z}$ displacements. The manipulator joystick performs five different functions: yaw, pitch, roll, open grip, and close grip. $\mathrm{X}$ and $\mathrm{Y}$ motions of the joystick are converted to pitch and yaw motions of the manipulator. Rotating the joystick performs the roll option and pressing button 1 or button 2 performs the close or open grip function. Figure 3 shows Saitek evo Force Feedback Joysticks.

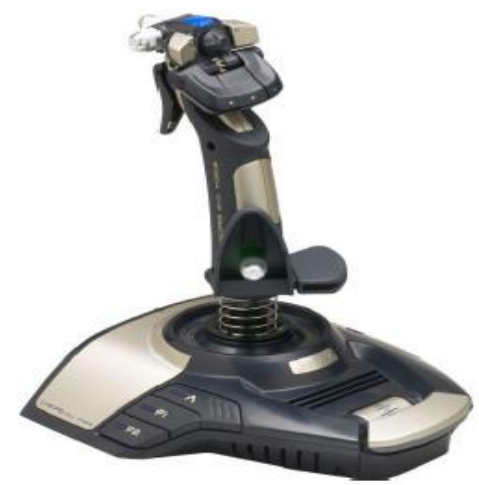

Figure 3. Saitek evo Force Feedback Joysticks 
The computer runs the systems software program which performs many functions but most importantly provides the user with the ability to control the robotic system. There are four different states of the program: Initialization, Trocar Placement, Insertion/Retraction, and Operation. The program starts up in the initialization state. The first task is to establish and verify communication with the Robotic Arm, dental Manipulator, and joysticks. Communication does not need to be established with the robot teach pendant since it is part of the robotic system. If communication is not established with any component, the program informs the user and then exits. This is done as a precaution so that all parts of the system are ready for operation before continuing. The second task in this state is to ask the user if the robot arm needs to be initialized. Performing this aligns the arm with the mathematical model of the arm stored in the controller's memory allowing the robot controller to know the position of the arm relative to the world. Initializing the robot can only be executed if the arm is in the homing bracket. This must be done if not previously executed since the robot controllers last power cycle. For safety the user of the system is notified of this action as opposed to automatic execution at the start-up of the program.

\section{Conventional Control (Second Order Sliding Mode Controller)}

Controller is a device which can sense information from linear or nonlinear system to improve the systems performance. The main targets in designing control systems are [11]:

- Stability

- good disturbance rejection

- small tracking error

Several industrial nonlinear plant are controlled by linear methodologies (e.g., Proportional-Derivative (PD) controller, Proportional- Integral (PI) controller or Proportional- Integral-Derivative (PID) controller), but when system works with various payloads and have uncertainty in dynamic models this technique has limitations.

From the control point of view, uncertainty is divided into two main groups [12]:

- Uncertainty in unstructured inputs (e.g., noise, disturbance)

- uncertainty in structure dynamics (e.g., payload, parameter variations)

In some applications multi-DOF actuators are used in an unknown and unstructured environment, therefore strong mathematical tools used in new control methodologies to design nonlinear robust controller with an acceptable performance (e.g., minimum error, good trajectory, disturbance rejection). Sliding mode controller is an influential nonlinear controller to certain and uncertain systems which it is based on system's dynamic model [14].

Sliding mode controller (SMC) is a powerful nonlinear controller which has been analyzed by many researchers especially in recent years. This theory was first proposed in the early 1950 by Emelyanov and several co-workers and has been extensively developed since then with the invention of high speed control devices [15]. The main reason to opt for this controller is its acceptable control performance in wide range and solves two most important challenging topics in control which names, stability and robustness. Sliding mode control theory for control joint of robot manipulator was first proposed in 1978 by Young to solve the set point problem $\left(\dot{\boldsymbol{q}}_{\boldsymbol{d}}=\mathbf{0}\right)$ by discontinuous method in the following form [16-19];

$\boldsymbol{\tau}_{(q, t)}= \begin{cases}\boldsymbol{\tau}_{\boldsymbol{i}}^{+}(\boldsymbol{q}, \boldsymbol{t}) & \text { if } \boldsymbol{S}_{\boldsymbol{i}}>0 \\ \boldsymbol{\tau}_{\boldsymbol{i}}^{-}(q, t) & \text { if } \boldsymbol{S}_{\boldsymbol{i}}<0\end{cases}$

where $\boldsymbol{S}_{\boldsymbol{i}}$ is sliding surface (switching surface), $\boldsymbol{i}=\mathbf{1}, \mathbf{2}, \ldots \ldots, \boldsymbol{n}$ for $n$-DOF joint, $\boldsymbol{\tau}_{\boldsymbol{i}}(\boldsymbol{q}, \boldsymbol{t})$ is the $\boldsymbol{i}^{\boldsymbol{t h}}$ torque of joint. Sliding mode controller is divided into two main sub controllers: 
- Corrective control $\left(\boldsymbol{U}_{\boldsymbol{c}}\right)$

- Equivalent controller $\left(\boldsymbol{U}_{\boldsymbol{e q}}\right)$.

Discontinues controller causes an acceptable tracking performance at the expense of very fast switching. Conversely in this theory good trajectory following is based on fast switching, fast switching is caused to have system instability and chattering phenomenon. Fine tuning the sliding surface slope is based on nonlinear equivalent part [20]. However, this controller is used in many applications but, pure sliding mode controller has two most important challenges: chattering phenomenon and nonlinear equivalent dynamic formulation in uncertain parameters[21-22].

Design a robust controller for multi-DOF-joints is essential because these joints have highly nonlinear dynamic parameters. Consider a nonlinear single input dynamic system is defined by:

$$
x^{(n)}=f(\vec{x})+b(\vec{x}) u
$$

Where $\mathrm{u}$ is the vector of control input, $\boldsymbol{x}^{(\boldsymbol{n})}$ is the $\boldsymbol{n}^{\text {th }}$ derivation of $\boldsymbol{x}, \boldsymbol{x}=$ $\left[\boldsymbol{x}, \dot{\boldsymbol{x}}, \ddot{\boldsymbol{x}}, \ldots, \boldsymbol{x}^{(\boldsymbol{n}-\mathbf{1})}\right]^{\boldsymbol{T}}$ is the state vector, $\boldsymbol{f}(\boldsymbol{x})$ is unknown or uncertainty, and $\boldsymbol{b}(\boldsymbol{x})$ is of known sign function. The main goal to design this controller is train to the desired state; $x_{d}=\left[x_{d}, \dot{x}_{d}, \ddot{x}_{d}, \ldots, x_{d}^{(n-1)}\right]^{T}$, and trucking error vector is defined by:

$$
\widetilde{x}=x-x_{d}=\left[\widetilde{x}, \ldots, \widetilde{x}^{(n-1)}\right]^{T}
$$

A simple solution to get the sliding condition when the dynamic parameters have uncertainty is the switching control law:

$$
U_{C}=\widehat{U}-K(\vec{x}, t) \cdot \operatorname{sgn}(s)
$$

where the switching function $\mathbf{s g n}(\mathbf{S})$ is defined as

$$
\operatorname{sgn}(s)= \begin{cases}1 & s>0 \\ -1 & s<0 \\ 0 & s=0\end{cases}
$$

and the $\boldsymbol{K}(\overrightarrow{\boldsymbol{x}}, \boldsymbol{t})$ is the positive constant.

Based on above discussion, the sliding mode control law for multi-DOF-joints is written as:

$$
\boldsymbol{U}=\boldsymbol{U}_{e q}+\boldsymbol{U}_{\boldsymbol{c}}
$$

where, the model-based component $\boldsymbol{U}_{\boldsymbol{e q}}$ is the nominal dynamics of systems and calculated as follows:

$$
U_{e q}=\left[H^{-1}(q)\left(B(q)\left[\begin{array}{c}
\dot{\alpha} \dot{\beta} \\
\dot{\alpha} \dot{\gamma} \\
\dot{\beta} \dot{\gamma}
\end{array}\right]+C(q)\left[\begin{array}{c}
\dot{\alpha}^{2} \\
\dot{\beta}^{2} \\
\dot{\gamma}^{2}
\end{array}\right]\right)+\dot{S}\right] H(q)
$$

and $\boldsymbol{U}_{\boldsymbol{c}}$ is computed as;

$$
\boldsymbol{U}_{\boldsymbol{c}}=\boldsymbol{K} \cdot \operatorname{sgn}(S)
$$

The sliding mode control of multi-DOF-joint is calculated as;

$$
\left[\begin{array}{c}
\widehat{\tau_{\alpha}} \\
\widehat{\boldsymbol{\tau}_{\beta}} \\
\widehat{\boldsymbol{\tau}_{\gamma}}
\end{array}\right]=\left[H^{-1}(\boldsymbol{q})\left(\mathrm{B}(\boldsymbol{q})\left[\begin{array}{c}
\dot{\alpha} \dot{\beta} \\
\dot{\alpha} \dot{\gamma} \\
\dot{\boldsymbol{\beta}} \dot{\gamma}
\end{array}\right]+C(q)\left[\begin{array}{c}
\dot{\alpha}^{2} \\
\dot{\beta}^{2} \\
\dot{\gamma}^{2}
\end{array}\right]\right)+\dot{S}\right] H(q)+K \cdot \operatorname{sgn}(S)
$$

The lyapunov formulation can be written as follows [22],

$$
V=\frac{1}{2} S^{T} . H . S
$$


the derivation of $V$ can be determined as,

$$
\dot{V}=\frac{1}{2} S^{T} \cdot \dot{H} \cdot S+S^{T} H \dot{S}
$$

the dynamic equation of multi-DOF actuator can be written based on the sliding surface as

$$
\boldsymbol{H} \dot{\boldsymbol{S}}=-\boldsymbol{V} \boldsymbol{S}+\boldsymbol{H} \dot{\boldsymbol{S}}+\boldsymbol{B}+\boldsymbol{C}
$$

it is assumed that

$$
S^{T}(\dot{H}-2 B+C) S=0
$$

by substituting (14) in (15)

$\dot{V}=\frac{1}{2} S^{T} \dot{H} S-S^{T} B+C S+S^{T}(H \dot{S}+B+C S)=S^{T}(H \dot{S}+B+C S)$

suppose the control input is written as follows

$$
\begin{aligned}
& \widehat{U}=\widehat{U_{\text {Nonlınear }}}+\widehat{U_{c}}=\left[\widehat{H^{-1}}(B+C)+\dot{S}\right] \widehat{H}+K \cdot \operatorname{sgn}(S)+B+C S \\
& \dot{V}=S^{T}\left(H \dot{S}+B+C-\widehat{H} \dot{S}-\widehat{B+C S}-K \operatorname{sgn}(S)=S^{T}(\widetilde{H} \dot{S}+\widehat{B+C S}-K \operatorname{sgn}(S))\right.
\end{aligned}
$$
and

$$
|\widetilde{\boldsymbol{H}} \dot{\boldsymbol{S}}+\widetilde{\boldsymbol{B + C}} \boldsymbol{S}| \leq|\widetilde{\boldsymbol{H}} \dot{\boldsymbol{S}}|+|\widetilde{\boldsymbol{B}+\boldsymbol{C}} \boldsymbol{S}|
$$

the Lemma equation in multi-DOF actuator can be written as follows

$$
K_{u}=[|\widetilde{H} \dot{S}|+|B+C S|+\eta]_{i}, i=1,2,3,4, \ldots
$$

and finally;

$$
\dot{V} \leq-\sum_{i=1}^{n} \eta_{i}\left|S_{i}\right|
$$

\section{Artificial Intelligence Control (Design Fuzzy Logic Control)}

The model-free control strategy is based on the assumption that the joints of the manipulators are all independent and the system can be decoupled into a group of singleaxis control systems. Therefore, the kinematic control method always results in a group of individual controllers, each for an active spherical joint. With the independent joint assumption, no a priori knowledge of spherical joint dynamics is needed in the kinematic controller design, so the complex computation of its dynamics can be avoided and the controller design can be greatly simplified. This is suitable for real-time control applications when powerful processors, which can execute complex algorithms rapidly, are not accessible. However, since joints coupling is neglected, control performance degrades as operating speed increases and a spherical joint controlled in this way is only appropriate for relatively slow motion. The fast motion requirement results in even higher dynamic coupling between the various spherical joint, which cannot be compensated for by a standard spherical joint controller such as PID, and hence model-based control becomes the alternative [13-16].

Based on fuzzy logic methodology

$$
f(x)=U_{f u z z y}=\sum_{l=1}^{M} \theta^{T} \zeta(x)
$$

where $\boldsymbol{\theta}^{\boldsymbol{T}}$ is adjustable parameter (gain updating factor) and $\boldsymbol{\zeta}(\boldsymbol{x})$ is defined by;

$$
\zeta(x)=\frac{\sum_{i} \mu\left(x_{i}\right) x_{i}}{\sum_{i} \mu\left(x_{i}\right)}
$$

Where $\boldsymbol{\mu}\left(\boldsymbol{x}_{\boldsymbol{i}}\right)$ is membership function. $\boldsymbol{\tau}_{\boldsymbol{f u z z} \boldsymbol{y}}$ is defined as follows; 


$$
\tau_{f u z z y}=\sum_{l=1}^{M} \theta^{T} \zeta(x)=\left[M^{-1}(B+C+G)+\dot{S}\right] M
$$

The main parts to design fuzzy controller are:

Fuzzification: the first step in fuzzification is determined inputs and outputs.

The second step is chosen an appropriate membership function for inputs and output. Membership function is used to solve a few problems:1) how to choose general parameters, such as the number of classes (membership functions) to describe all the values of the linguistic variable on the universe, the position of different membership functions on the universe of discourse, the width of the membership functions, and concrete parameters, such as the shape of a particular membership function.

The third step is chosen the correct labels for each fuzzy set.

Fuzzy Rule Base and Rule Evaluation: the first step in rule base and evaluation is to provide a least structured method to derive the fuzzy rule base which, expert experience and control engineering knowledge is used because this method is the least structure of the other one and the researcher derivation the fuzzy rule base from the knowledge of system operate and/or the classical controller. Rule evaluation focuses on operation in the antecedent of the fuzzy rules in proposed method.

Aggregation of the Rule Output (Fuzzy Inference): There are several methodologies in aggregation of the rule outputs and used in fuzzy logic controllers, namely,

- Max-Min aggregation

- Sum-Min aggregation

- Max-bounded product

- Max-drastic product

- Max-bounded sum

- Max-algebraic sum

- Min-max

Two most common methods that used in fuzzy logic controllers are Max-min aggregation and Sum-min aggregation. Max-min aggregation defined as below

$$
\mu_{U}\left(x_{k}, y_{k}, U\right)=\mu_{\cup_{i=1}^{r} F R^{i}}\left(x_{k}, y_{k}, U\right)=\max \left\{\min _{i=1}^{r}\left[\mu_{R}{ }_{p q}\left(x_{k}, y_{k}\right), \mu_{p_{m}}(U)\right]\right\}
$$

The Sum-min aggregation defined as below

$$
\mu_{U}\left(x_{k}, y_{k}, U\right)=\mu_{\cup_{i=1}^{r} F R^{i}}\left(x_{k}, y_{k}, U\right)=\sum \min _{i=1}^{r}\left[\mu_{R p q}\left(x_{k}, y_{k}\right), \mu_{p_{m}}(U)\right]
$$

where $r$ is the number of fuzzy rules activated by $x_{k}$ and $y_{k}$ and also $\mu_{\cup_{i=1}^{r} F R^{i}}\left(x_{k}, y_{k}, U\right)$ is a fuzzy interpretation of $i-t h$ rule. Max-Min aggregation is used in this work.

Defuzzification: The last step in the fuzzy inference in any fuzzy set is defuzzification. This part is used to transform fuzzy set to crisp set, therefore the input for defuzzification is the aggregate output and the output is a crisp number. There are several methodologies in defuzzification of the rule outputs that can be used in fuzzy logic controllers but two most common defuzzification methods are: Center of gravity method $(C O G)$ and Center of area method $(C O A)$, which $C O G$ method used the following equation to calculate the defuzzification

$$
\operatorname{COG}\left(x_{k}, y_{k}\right)=\frac{\sum_{i} U_{i} \sum_{j=1}^{r}, \mu_{u}\left(x_{k}, y_{k}, U_{i}\right)}{\sum_{i} \sum_{j=1}^{r}, \mu_{u}\left(x_{k}, y_{k}, U_{i}\right)}
$$


and $C O A$ method used the following equation to calculate the defuzzification

$$
\operatorname{COA}\left(x_{k}, y_{k}\right)=\frac{\sum_{i} U_{i}, \mu_{u}\left(x_{k}, y_{k}, U_{i}\right)}{\sum_{i} \mu_{U} \cdot\left(x_{k}, y_{k}, U_{i}\right)}
$$

Where $\operatorname{COG}\left(x_{k}, y_{k}\right)$ and $\operatorname{COA}\left(x_{k}, y_{k}\right)$ illustrates the crisp value of defuzzification output, $U_{i} \in U$ is discrete element of an output of the fuzzy set, $\mu_{U} .\left(x_{k}, y_{k}, U_{i}\right)$ is the fuzzy set membership function, and $r$ is the number of fuzzy rules. Center of gravity method $(C O G)$ is used in this research.

\section{Methodology: Design Limit Chatter/Oscillation Control}

To limit the chattering, various papers have been reported by many researchers. In this research intelligent method is used to reduce/eliminate the chattering in presence of uncertainty with respect to improve the robustness. To control/limit the chattering fuzzy logic controller is used and applied to conventional SMC. This type of controller has double inputs and an output.

Fuzzy control emerged on the foundations of Zadeh's fuzzy set theory. It is a methodology of intelligent control that mimics human thinking and reacting by using a multivalent fuzzy logic and elements of artificial intelligence (simplified deduction principles). The word "fuzzy" is used here to describe terms that are either not wellknown or not clear enough, or their closer specification depends on subjectivity, estimation, and even the intuition of the person who is describing these terms. In everyday life there are a lot of situations characterized by a certain degree of ambiguity whose description includes terms and expressions such as majority, many, several, not exactly, or quite possible, all of which can be qualified as "fuzzy terms." On the other hand, terms like false, true, possible, necessary, none, or all reflect crisp meanings, and in such a context, represent "exact terms." The fuzzy logic concept had very strong opponents in the beginning. They believed that any form of vagueness or imprecision could be equally well described with the theory of probability. Furthermore, opponents claimed that fuzzy logic theory was only a theory without real potential for practical applications. In the field of automatic control, the strongest opponents assumed that traditional control techniques were superior to fuzzy logic or at least equal in effect. The kind of a structure a fuzzy controller will have will primarily depend on the controlled process and the demanded quality of control. Since the application area for fuzzy control is really wide, there are many possible controller structures, some differing significantly from each other by the number of inputs and outputs, or less significantly by the number of input and output fuzzy sets and their membership functions forms, or by the form of control rules, the type of inference engine, and the method of defuzzification. All that variety is at the designer's disposal, and it is up to the designer to decide which controller structure would be optimal for a particular control problem. For example, if the controlled process exhibits integral behavior, then a so-called non-integral or PD-type fuzzy controller whose crisp output value represents absolute control input value could provide the required quality of control. On the other hand, a so-called integral or PI-type fuzzy controller whose crisp output value represents an increment of control input value could be a satisfactory solution for the control of static systems. The usage of fuzzy algorithms is not limited to fuzzy logic controllers. Fuzzy algorithms can be used equally well as nonlinear adaptation mechanisms, universal approximators or as auxiliary units added to some conventional control solutions. We should not fail to mention that fuzzy controllers are very convenient as supervisory controllers. Sometimes, fuzzy logic algorithms are also used as modal or fuzzy state controllers. Despite the variety of possible fuzzy controller structures, the basic form of all common types of controllers consists of:

- Input fuzzification (binary-to-fuzzy[B/F]conversion) 
- Fuzzy rule base (knowledge base)

- Inference engine

- Output defuzzification (fuzzy-to-binary [F/B] conversion).

The most frequently used structure of a fuzzy controller is the double input-single output (DISO) structure. In case of designing such a controller, a very convenient form of displaying the complete fuzzy rule base is a fuzzy rule table. Every rule in the fuzzy rule table is represented by an output fuzzy set engaged in the THEN part of the rule. The rule position within the fuzzy rule Table is determined by coordinates of inputs fuzzy sets engaged in the IF part of the rule. Thus the fuzzy rule table provides straight insight into the essence of the fuzzy rule base and automatically eliminates the creation of contradictory fuzzy rules. The tabular format also makes an elegant entry of new fuzzy rules possible.

Table 1 shows the fuzzy rule-Table of a DISO fuzzy controller with $l=5$ triangular fuzzy sets defined for both inputs $x$ (error) and $y$ (integral of error), and output $u$ as following: negative large (NL); negative small (NS); around zero (Z); positive small (PS); and positive large (PL). For the studied $l \times l=5 \times 5$ table a number of fuzzy rules may increase up to $l^{2}=25$ rules.

Table 1. A Fuzzy Rule Base Displayed as a Fuzzy Rule Table

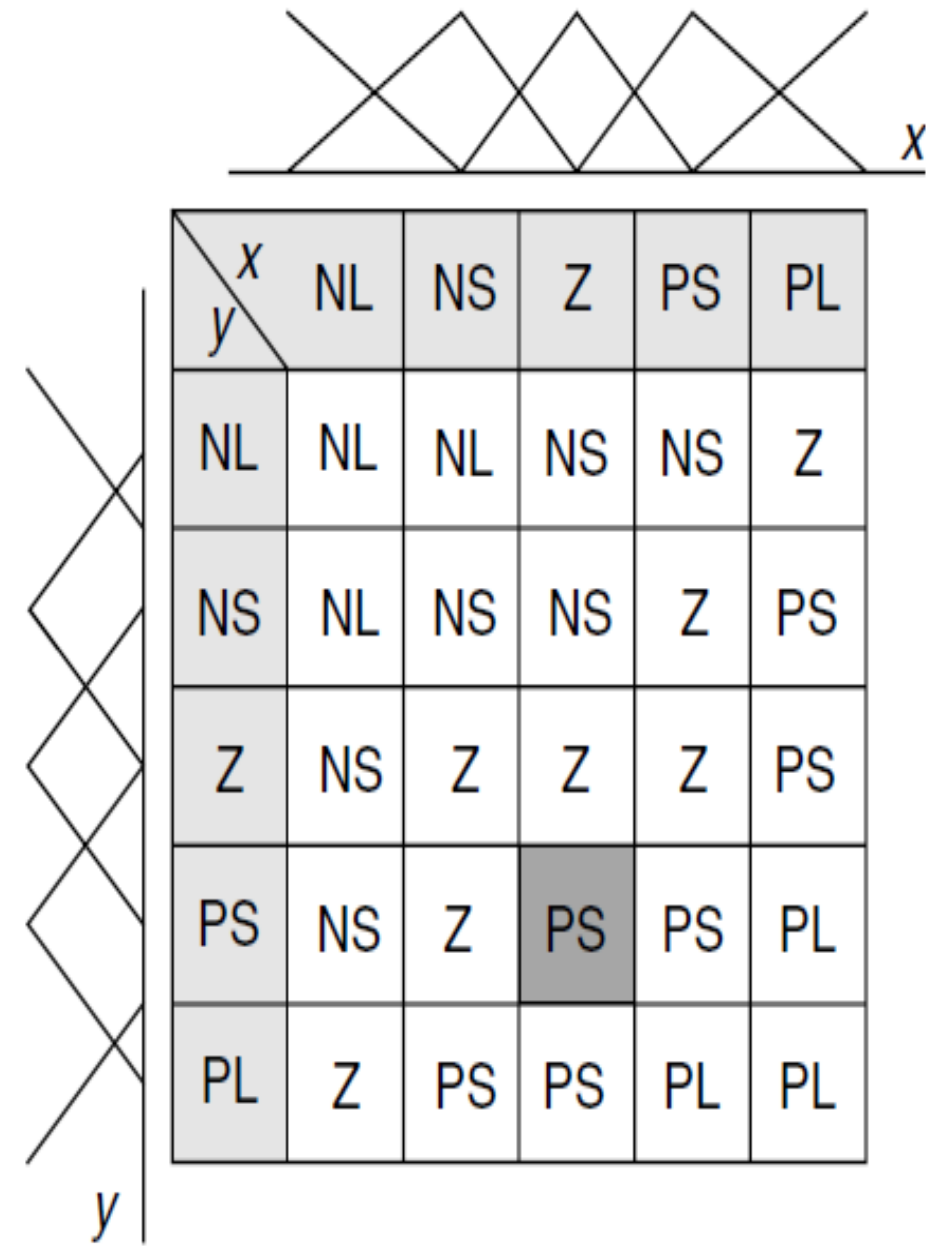

The shaded rule in Table 1 can be read as follows:

IF $x$ is Z AND $y$ is PS THEN $u$ is PS 
A short glance at the table confirms the completeness (all 25 rules are there) and the continuity of the displayed fuzzy rule-base (consistency is automatically provided). A fuzzy rule table can also be viewed as the state space of two process variables $x$ and $y$ (e.g., let $x=e(k)$ - control error, $\Delta y_{f}=$ Derivative of $x$ - change of error $(\dot{e})$, where $k$ is the substitute for $k T_{d}$, and $T_{d}$ is a sampling interval). By using a fuzzy rule Table, we get the chance to see the corresponding phase trajectories resulting from consecutive switching of fuzzy rules (Table 2).

Table 2. Phase Trajectories Drawn in a Fuzzy Rule Table

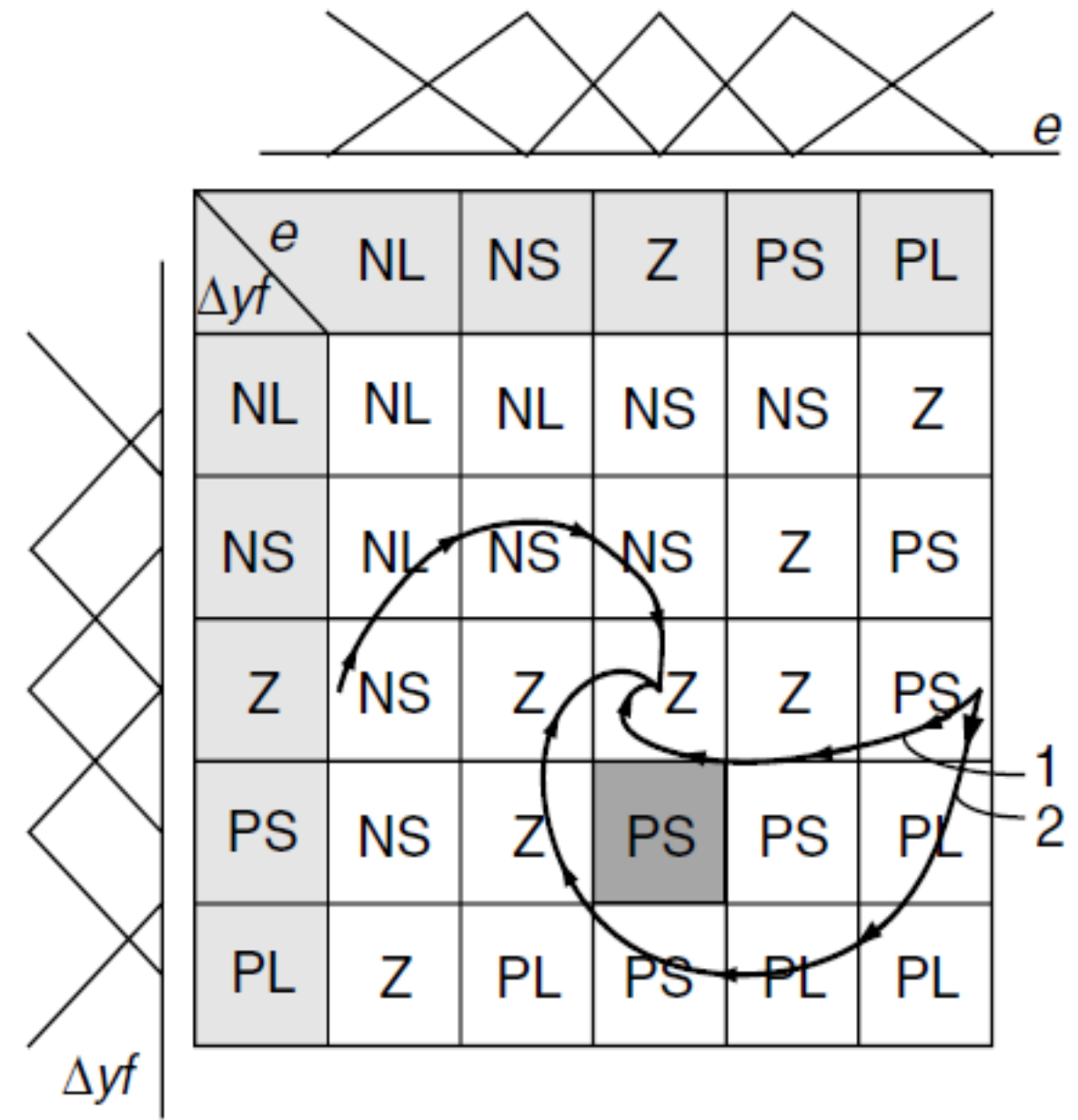

We have already mentioned that the design of a fuzzy controller is actually a heuristic search for the best fitted static nonlinear mapping function between controller inputs and outputs. As a result of mapping, every discrete trajectory $\left[e(k), y_{f(k)}\right]$ has a matching controller output series $u_{F C(k)}, k=0,1, \ldots, \infty$ space composed of a phase plane and a corresponding fuzzy control surface lying above the plane. Every controller output sequence $u_{F C(k)}$ belongs to this fuzzy control surface. Any changes made in the fuzzy rule table during the design process will change the path of phase trajectories. Therefore, these trajectories are very useful for getting a better insight into the progress of an ongoing controller design. By following the trajectory during a transient response one can easily find which fuzzy control rules are activated and how they contribute to crisp output value. A fuzzy rule table viewed as a phase plane is frequently used for heuristic assessment of closed-loop system stability, as it offers an elegant way to investigate the 
influence of individual control rules (their THEN parts) on the shape of phase trajectories. In order to bring more generality into the process of controller design, we would advise normalization of controller input and output domains. The universe of discourse of fuzzy controller inputs and outputs varies from one application to another. To avoid having to make adjustments for each application, inputs and outputs can be scaled to fit the normalized universes of discourse. When we use the term normalized fuzzy controller, we have in mind a controller whose fuzzification, fuzzy rule base and defuzzification parts operate with normalized values usually lying in the interval $[-1,1]$.

The normalization of inputs should be performed before proceeding with fuzzification:

$$
x_{N}(k)=K_{x} x(k)
$$

where $x$ is controller input, $\boldsymbol{x}_{\boldsymbol{N}}$ is normalized controller input, and $\boldsymbol{K}_{\boldsymbol{x}}$ is the scaling factor. Thus, $e(k)$ and $\sum e(k)$ after normalization become:

$$
\begin{aligned}
& \boldsymbol{e}_{\boldsymbol{N}}(\boldsymbol{k})=\boldsymbol{K}_{\boldsymbol{e}} \boldsymbol{e}(\boldsymbol{k}) \\
& \sum \boldsymbol{e}_{\boldsymbol{N}}(\boldsymbol{k})=\boldsymbol{K}_{\sum e} \sum \boldsymbol{e}(\boldsymbol{k})
\end{aligned}
$$

After limitation the chattering based on DISO fuzzy controller, the second SISO fuzzy controller is used to data tracking in uncertain condition. No matter how complicated the control of a plant may seem, the majority of control loops in industrial control systems utilize standard P, PI, PD, or PID control algorithms (here denoted as P-I-D) with fixed parameter values set during the commissioning. The synthesis of P-I-D controller parameters based on well-known design methods normally requires a mathematical model, which can precisely describe the dynamical behavior of a control object. Values of P-I-D controller parameters obtained in such a way describe a linear control law adequate for a selected operating point. If such a controller is applied to a nonlinear control system, the performance of the system will vary depending on the variations of control object parameters. In addition, the usage of a linear control law will cause different responses of a nonlinear system for the same magnitude of positive and negative reference input changes.

Different design strategies have been developed with the purpose to overcome the disadvantages of linear P-I-D controllers. Such strategies transform a linear P-I-D controller into P-I-D-like structures of fuzzy controllers PD+I. When designing a fuzzy controller by emulating of a linear PD+I controller, we assume that the fuzzy controller should inherit the linear character of its model. In order to evaluate the quality of such a transformation, different measures of achieved linearity have been introduced. In terms of the influence that different fuzzy reasoning methods (fuzzy implications) have on the achieved linearity of PD+I-like fuzzy controllers, theoretical results show that the vast majority of fuzzy PD+I controller is actually nonlinear PD+I controller. In this research it has been proven that PD+I controllers are nonlinear PD+I controllers with P-gain, Igain, and D-gain changing with the output of the controlled system, providing that they have five triangular input fuzzy sets for each input variable, fuzzy rules with a triangular in the consequent part, Zadeh's AND operator and the centroid defuzzifier. Although the making a perfect fuzzy copy of a linear PD+I controller could be an interesting design goal, it is more important to use a linear PD+I controller as a starting point for the initial setting of a fuzzy controller, because its prime role is not to mimic the original, but to use all of the original's intrinsic nonlinear control potential. This can be achieved through various adaptive and self-organizing (self-learning) design concepts. When a more general solution is wanted, then phase space and phase plane are utilized.

A PD+I controller has the following form in continuous time domain:

$$
\boldsymbol{u}(\boldsymbol{t})=\left(\boldsymbol{K}_{p} \boldsymbol{e}+\boldsymbol{K}_{\boldsymbol{D}} \dot{\boldsymbol{e}}\right)+\boldsymbol{K}_{i} \sum \boldsymbol{e}
$$

Then the output of the SISO fuzzy controller gets the form: 


$$
e_{F}(k)=\sum_{\boldsymbol{l}=\mathbf{1}}^{\boldsymbol{M}} \boldsymbol{\theta}^{\boldsymbol{T}} \boldsymbol{\zeta}(\boldsymbol{x})
$$

$\boldsymbol{\theta}^{\boldsymbol{T}}$ is adjustable parameter and $\boldsymbol{\zeta}(\boldsymbol{x})$ is;

$$
\zeta(x)=\frac{\sum_{i} \mu\left(x_{i}\right) x_{i}}{\sum_{i} \mu\left(x_{i}\right)}
$$

$\boldsymbol{\mu}\left(\boldsymbol{x}_{\boldsymbol{i}}\right)$ is membership function.

\section{Results}

In this part two types of controller are compared, namely; conventional sliding mode controller, and intelligent chatter limiter sliding mode controller. These controllers are tested in certain and uncertain situation.

Comparison of the Tracking Data and Information: the trajectory following of 3 DOF joint for conventional sliding mode controller and intelligent chatter limiter sliding mode controller are compared in this part. According to Figure 4, traditional sliding mode controller and intelligent chatter limiter sliding mode controller have high frequency oscillation chattering phenomenon, however these controllers are robust.

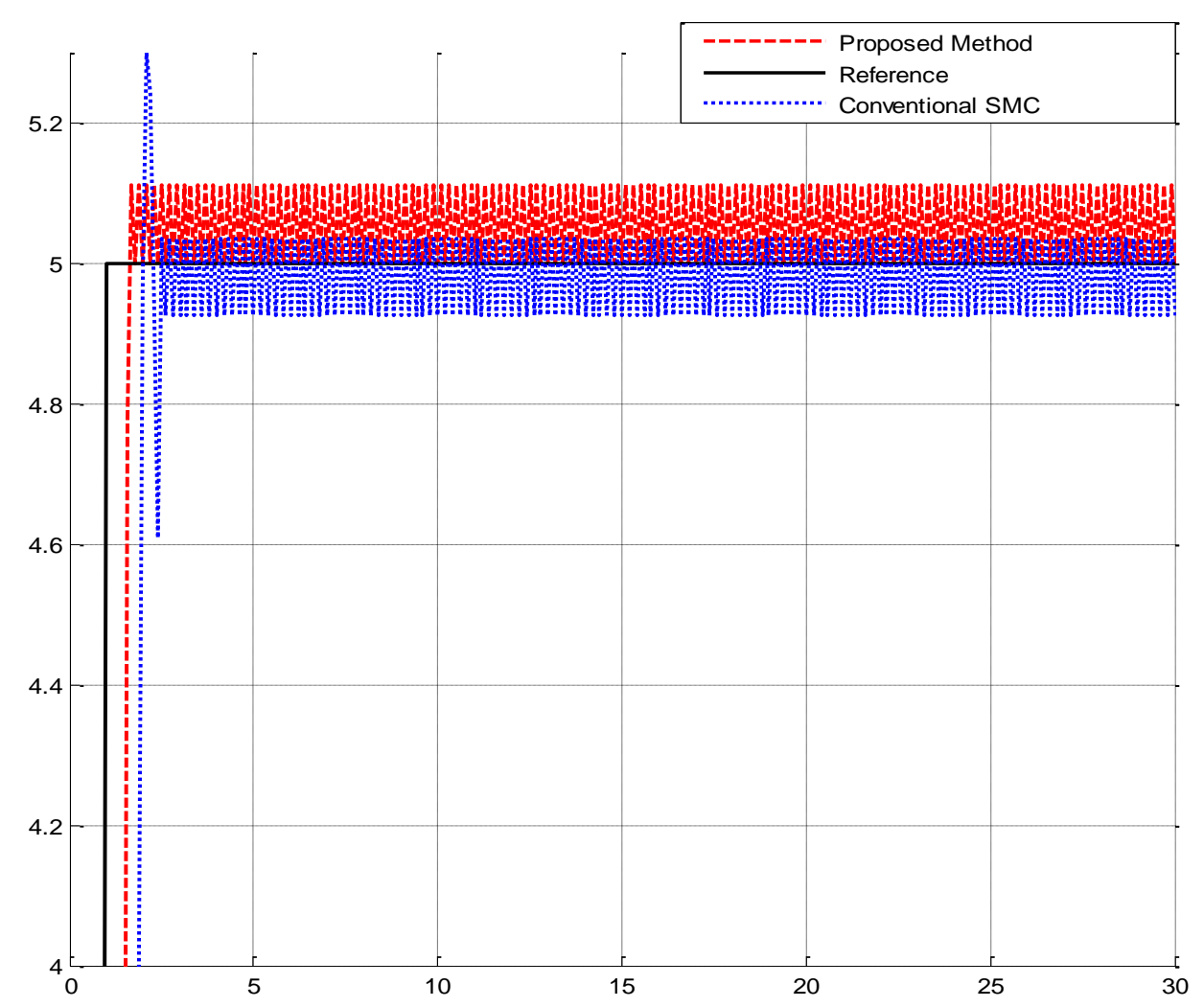

Figure 4. Trajectory Tracking Control

Regarding to Figure 4, conventional SMC has about $6 \%$ overshoot and it has more rise time than proposed methodology.

Comparison the Actuation Torque $\left(\boldsymbol{\tau}_{\boldsymbol{i}}\right)$ : the control input, forces the multi DOF joint to track the desired trajectories. Figure 5 shows the torque performance in conventional sliding mode controller and intelligent chatter limiter sliding mode controller. 


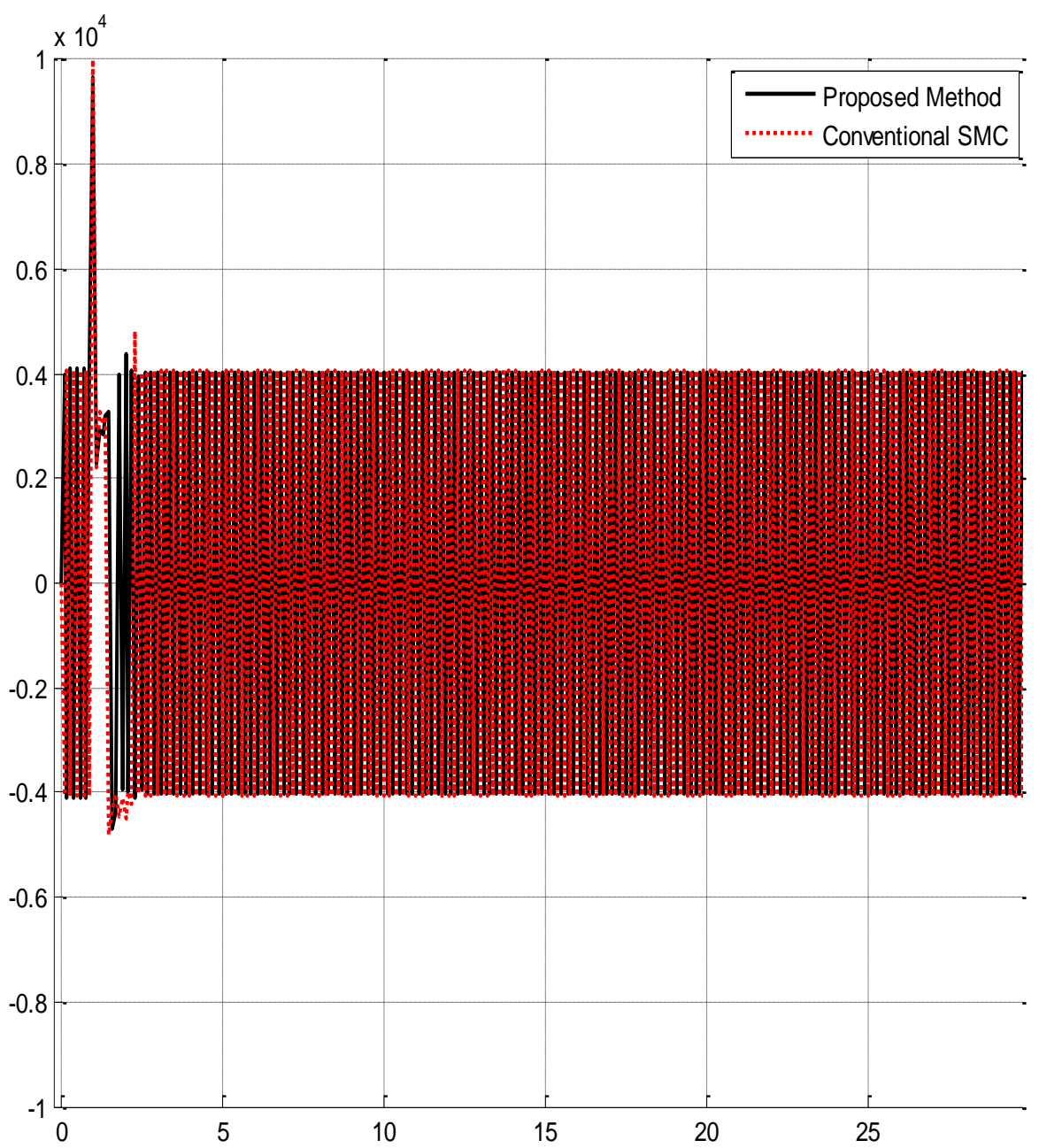

Figure 5. Torque Performance: Conventional SMC and Intelligent Chatter Limiter Sliding Mode Controller

Comparison the Disturbance Rejection: the power of disturbance rejection is very important to robust checking in any controllers. In this section trajectory accuracy, is test under uncertainty condition. To test the disturbance rejection band limited white noise with $70 \%$ amplitude is applied to conventional sliding mode controller and intelligent chatter limiter sliding mode controller. Figure 6 shows the power of disturbance rejection in conventional SMC and intelligent chatter limiter sliding mode controller. Regarding to the following Figure proposed method is more robust than conventional SMC in presence of uncertainty. 




Figure 6. Tracking Data: Conventional SMC and Intelligent Chatter Limiter Sliding Mode Controller in Presence of Uncertainty

\section{Conclusion}

The application of artificial intelligence such as neural networks and fuzzy logic in vibration control of dental robot arm investigated in this research. Analytical dynamic nonlinear modelling of the plant is carried out using elegant Euler-Lagrange method compromising accuracy and complexity. The main targets in this research are; stability, good disturbance rejection, and small tracking error. Several dental robot manipulators are controlled by linear methodologies (e.g., Proportional-Derivative (PD) controller, Proportional- Integral (PI) controller or Proportional- Integral-Derivative (PID) controller), but when dental robot manipulator works with various payloads and have uncertainty ${ }_{2}$ this technique has limitations. In dental applications multi DOF joint manipulators are used in unknown and unstructured environment, therefore strong mathematical tools used in new control methodologies to design nonlinear robust controller.

Sliding mode technique is used to control multi DOF joint manipulators, because it is robust and stable. However, pure Variable Structure controller is used in many applications; it has two important challenges; chattering phenomenon (high frequency vibration), and nonlinear equivalent dynamic formulation. In this research the chattering phenomenon is positive factor. Therefore this research is used to control (not reduce/ not eliminate) the chattering based on design fuzzy $(\mathrm{PD}+\mathrm{I})^{2}$ control techniques for conventional sliding mode controller. However, sliding mode controller is stable but in highly uncertain condition, it has many challenges, proposed method control the amplitude of chattering in presence of uncertainty, and controls the system's vibration. 


\section{Acknowledgment}

The authors would like to thank the anonymous reviewers for their careful reading of this paper and for their helpful comments. This work was supported by the Iranian Institute of Advance Science and Technology Program of Iran under grant no. 2013Persian Gulf-2A.

\section{Project Title: "Design High Precision and Fast Dynamic Controller For Multi- Degrees of Freedom Actuator"}

Iranian center of Advance Science and Technology (IRAN SSP) is one of the independent research centers specializing in research and training across of Control and Automation, Electrical and Electronic Engineering, and Mechatronics \& Robotics in Iran. At IRAN SSP research center, we are united and energized by one mission to discover and develop innovative engineering methodology that solve the most important challenges in field of advance science and technology. The IRAN SSP Center is instead to fill a long standing void in applied engineering by linking the training a development function one side and policy research on the other. This center divided into two main units:

- $\quad$ Education unit

- $\quad$ Research and Development unit

\section{References}

[1] R. A. Freitas Jr., "Nanodentistry", Journal of American Dental Association, vol. 131, no. 11, pp. 155965.

[2] J. L. West and N. J. Halas, "Applications of nanotechnology to biotechnology commentary", Curr Opin Biotechnol, vol. 11, (2000), pp. 215-7.

[3] H. Shi, W. B. Tsai, M. D. Garrison, S. Ferrari and B. D. Ratner, "Templateimprinted nanostructured surfaces for protein recognition", Nature, vol. 398, (1999), pp. 593-7.

[4] M. R. Sims, "Brackets, epitopes and flash memory cards: a futuristic view of clinical orthodontics", Aust Orthod J., vol. 15, (1999), pp. 260-8.

[5] H. C. Slavkin, "Entering the era of molecular dentistry", J Am Dent Assoc., vol. 130, (1999) March, pp. 413-7.

[6] T. R. Kurfess, "Robotics and automation handbook", CRC, (2005).

[7] L. Cheng, Z. G. Hou, M. Tan, D. Liu and A. M. Zou, "Multi-agent based adaptive consensus control for multiple manipulators with kinematic uncertainties", (2008), pp. 189-194.

[8] B. Siciliano and O. Khatib, Springer handbook of robotics: Springer-Verlag New York Inc, (2008).

[9] J. Wang, A. Rad and P. Chan, "Indirect adaptive fuzzy sliding mode control: Part I: fuzzy switching", Fuzzy Sets and Systems, vol. 122, no. 1, (2001), pp. 21-30.

[10] C. Wu, "Robot accuracy analysis based on kinematics," IEEE Journal of Robotics and Automation, vol. 2, no. 3, (1986), pp. 171-179.

[11] B. K. Yoo and W. C. Ham, "Adaptive control of robot manipulator using fuzzy compensator," Fuzzy Systems, IEEE Transactions on, vol. 8, no. 2, (2002), pp. 186-199.

[12] F. Piltan, S. H. T. Haghighi, N. Sulaiman, I. Nazari and S. Siamak, "Artificial Control of PUMA Robot Manipulator: A-Review of Fuzzy Inference Engine and Application to Classical Controller", International Journal of Robotics and Automation, vol. 2, no. 5, (2011), pp. 401-425.

[13] L. A. Zadeh, "Toward a theory of fuzzy information granulation and its centrality in human reasoning and fuzzy logic", Fuzzy Sets and Systems, vol. 90, no. 2, (1997), pp. 111-127.

[14] L. Reznik, Fuzzy controllers: Butterworth-Heinemann, (1997).

[15] J. Zhou and P. Coiffet, "Fuzzy control of robots," IEE proceeding Control Theory and Applications, vol. 147, no. 2, (2002), pp. 1357-1364.

[16] S. Banerjee and P. Y. Woo, "Fuzzy logic control of robot manipulator," Second IEEE conference on Control Applications, (2002), pp. 87-88.

[17] K. Kumbla, E. Tunstel and M. Jamshidi, "Soft computing for autonomous robotic systems," Computers and Electrical Engineering, vol. 26, no. 1, (2000), pp. 5-32.

[18] C. C. Lee, "Fuzzy logic in control systems: fuzzy logic controller. I," IEEE Transactions on systems, man and cybernetics, vol. 20, no. 2, (1990), pp. 404-418. 
[19] M. Mirshekaran, F. Piltan, N. Sulaiman, A. Salehi, M. Kazeminasab and Z. Esmaeili, "Hand Tremors Reduction Based on Integral Intelligent Filter Computed Torque Controller", International Journal of Bio-Science and Bio-Technology, vol. 7, no. 1, (2015), pp. 105-120, http://dx.doi.org/10.14257/ijbsbt.2015.7.1.11. (Scopus, SJR=0.24, Q3).

[20] M. Vosoogh, F. Piltan, A. M. Mirshekaran, A. Barzegar, A. Siahbazi and N. Sulaiman, "Integral Criterion-Based Adaptation Control to Vibration Reduction in Sensitive Actuators", International Journal of Hybrid Information Technology, vol. 8, no. 2, (2015), pp. 11-30, http://dx.doi.org/10.14257/ijhit.2015.8.2.02.

[21] M. Yaghoot, F. Piltan, M. Esmaeili, M. A. Tayebi and M. Piltan, "Design Intelligent Robust Modelbase Sliding Guidance Controller for Spherical Motor", IJMECS, vol. 6, no. 3, (2014), pp. 61-72, DOI: 10.5815/ijmecs.2014.03.08.

[22] F. Matin, F. Piltan, H. Cheraghi, N. Sobhani and M. Rahmani, "Design Intelligent PID like Fuzzy Sliding Mode Controller for Spherical Motor", IJIEEB, vol. 6, no. 2, (2014), pp. 53-63, DOI: 10.5815/ijieeb.2014.02.07.

\section{Authors}

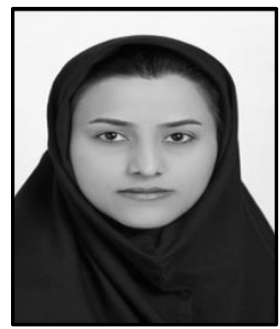

Maryam Rahmani, currently is research assistant at Iranian Institute of Advanced Science and Technology, IRAN SSP. She is research assistant of team (8 researchers) to design "Micro-electronic Based intelligent nonlinear controller" since Feb, 2015 to Aug, 2015, research student (21 researchers) to design high precision and fast dynamic controller for multi-degrees of freedom actuator since July, 2013 to July, 2014, and published 5 journal papers since 2013 to date. Her current research interests are nonlinear control, artificial control system, Microelectronic Device, and HDL design. (http://iranssp.org/english/?page_id=2144).



Nasim Sobhani, has been working at "Design High Precision and Fast Dynamic Controller for Multi-Degrees of Freedom Actuator for Experimental Research and Education" project at Iranian Institute of Advance Science and Technology, Sanaat Kade Sabz Passargad Research Center (IRAN SSP) as "Student researcher "of a research team composed of 21 researchers since July 2013 to July 2014.

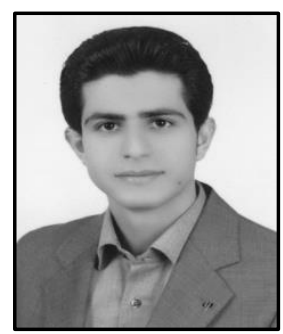

Hamid Cheraghi, currently is research assistant at Iranian Institute of Advanced Science and Technology, IRAN SSP. He is research assistant of team (8 researchers) to design "Micro-electronic Based intelligent nonlinear controller" since Feb, 2015 to Aug, 2015, research student (21 researchers) to design high precision and fast dynamic controller for multi-degrees of freedom actuator since July, 2013 to July, 2014, and published 5 journal papers since 2013 to date. Her current research interests are nonlinear control, artificial control system, Microelectronic Device, and HDL design. (http://iranssp.org/english/?page_id=2144). 




Farzin Piltan, was born on 1975, Shiraz, Iran. In 2004 he is jointed Institute of Advance Science and Technology, Research and Development Center, IRAN SSP. Now he is a dean of Intelligent Control and Robotics Lab. He is led of team (47 researchers) to design and build of nonlinear control of industrial robot manipulator for experimental research and education and published about 54 Papers in this field since 2010 to 2012, team supervisor and leader (9 researchers) to design and implement intelligent tuning the rate of fuel ratio in internal combustion engine for experimental research and education and published about 17 Journal papers since 2011 to 2013 , team leader and advisor (34 researchers) of filtering the hand tremors in flexible surgical robot for experimental research and education and published about 31 journal papers in this field since 2012 to date, led of team ( 21 researchers) to design high precision and fast dynamic controller for multi-degrees of freedom actuator for experimental research and education and published about 7 journal papers in this field since 2013 to date, led of team ( 22 researchers) to research of full digital control for nonlinear systems (e.g., Industrial Robot Manipulator, IC Engine, Continuum Robot, and Spherical Motor) for experimental research and education and published about 4 journal papers in this field since 2010 to date, team team supervisor and leader to design Intelligent FPGA-Based Control Unit to Control of 4-DOF Medical Robot Manipulator since July, 2015 to now, team supervisor and leader of team (8 researchers) to design a Microelectronic Based nonlinear controller for first order delay system since March, 2015 to now, team supervisor and leader (4 researchers) to design Intelligent Vibration Robot control for Dental Automation since Jun, 2014 to now and finally led of team (more than 130 researchers) to implementation of Project Based-Learning project at IRAN SSP research center for experimental research and education, and published more than 115 journal papers since 2010 to date. In addition to 7 textbooks, Farzin Piltan is the main author of more than 115 scientific papers in refereed journals. He is editorial review board member for 'international journal of control and automation (IJCA), Australia, ISSN: 2005-4297; 'International Journal of Intelligent System and Applications (IJISA)', Hong Kong, ISSN:2074-9058; 'IAES international journal of robotics and automation, Malaysia, ISSN:2089-4856; 'International Journal of Reconfigurable and Embedded Systems', Malaysia, ISSN:20894864. His current research interests are nonlinear control, artificial control system and applied to FPGA, robotics and artificial nonlinear control and IC engine modeling and control.

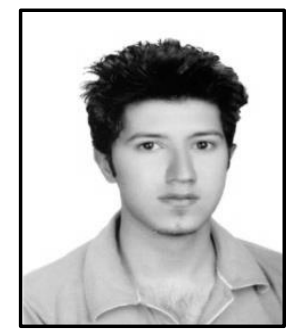

Farzin Matin, has been working at "Design High Precision and Fast Dynamic Controller for Multi-Degrees of Freedom Actuator for Experimental Research and Education" project at Iranian Institute of Advance Science and Technology, Sanaat Kade Sabz Passargad Research Center (IRAN SSP) as "Student researcher " of a research team composed of 21 researchers since July 2013 to July 2014. 
International Journal of Hybrid Information Technology

Vol.8, No.10 (2015) 